\title{
Penile Glomus Tumor
}

National Cancer Institute

\section{Source}

National Cancer Institute. Penile Glomus Tumor. NCI Thesaurus. Code C162582.

A glomus tumor that occurs in the penis. 